\title{
Wolbachia infections in native and introduced populations of fire ants (Solenopsis spp.)
}

\author{
D. DeWayne Shoemaker, ${ }^{1}$ Kenneth G. Ross, ${ }^{2}$ \\ L. Keller, ${ }^{3}$ E. L. Vargo ${ }^{4}$ and John H. Werren ${ }^{5}$ \\ ${ }^{1}$ Department of Biological Sciences, 3149 Wood Hall, \\ 1903 West Michigan Avenue, Western Michigan \\ University, Kalamazoo, MI 49008-5410, USA, \\ ${ }^{2}$ Department of Entomology, University of Georgia, \\ Athens, GA 30602-2603, USA, ${ }^{3}$ University of Lausanne, \\ Institute of Ecology, Batiment de Biologie, 1015 \\ Lausanne, Switzerland, ${ }^{4}$ Department of Entomology, \\ Campus Box 7613, North Carolina State University, \\ Raleigh, NC, 27695-7613, USA, ${ }^{5}$ Department of Biology, \\ University of Rochester, Rochester, NY 14627-0211, USA
}

\begin{abstract}
Wolbachia are cytoplasmically inherited bacteria that induce a variety of effects with fitness consequences on host arthropods, including cytoplasmic incompatibility, parthenogenesis, male-killing and feminization. We report here the presence of Wolbachia in native South American populations of the fire ant Solenopsis invicta, but the apparent absence of the bacteria in introduced populations of this pest species in the USA. The Wolbachia strains in native $S$. invicta are of two divergent types ( $A$ and $B$ ), and the frequency of infection varies dramatically between geographical regions and social forms of this host. Survey data reveal that Wolbachia also are found in other native fire ant species within the Solenopsis saevissima species complex from South America, including S. richteri. This latter species also has been introduced in the USA, where it lacks Wolbachia. Sequence data reveal complete phylogenetic concordance between mtDNA haplotype in S. invicta and Wolbachia infection type (A or B). In addition, the mtDNA and associated group A Wolbachia strain in S. invicta are more closely related to the mtDNA and Wolbachia strain found in S. richteri than they are to the mtDNA and associated group $B$
\end{abstract}

Received 16 May 2000; accepted after revision 24 August 2000. Correspondence: D. DeWayne Shoemaker, Department of Biological Sciences, 3149 Wood Hall, 1903 West Michigan Avenue, Western Michigan University, Kalamazoo, MI 49008-5410, USA. Tel.: 616387 2084; fax: 616 387 5609; e-mail: dewayne.shoemaker@wmich.edu
Wolbachia in S. invicta. These data are consistent with historical introgression of $S$. richteri cytoplasmic elements into $S$. invicta populations, resulting in enhanced infection and mtDNA polymorphisms in S. invicta. Wolbachia may have significant fitness effects on these hosts (either directly or by cytoplasmic incompatibility) and therefore these microbes potentially could be used in biological control programmes to suppress introduced fire ant populations.

Keywords: biological control, fire ants, mtDNA, introgression, reproductive isolation, Solenopsis invicta, Wolbachia.

\section{Introduction}

Wolbachia are maternally transmitted alpha-proteobacteria that infect the various tissues of arthropods, and whose transmission is enhanced by a variety of mechanisms including cytoplasmic incompatibility, thelytokous parthenogenesis, feminization of genetic males, male-killing, and increased mating success of infected males via sperm competition (Beard et al., 1993; Breeuwer et al., 1992; Hurst et al., 1999; O'Neill et al., 1992; Sinkins et al., 1995a,b; Stouthamer et al., 1993; Wade \& Chang, 1995; for recent reviews see Stouthamer et al., 1999; Werren \& O'Neill, 1997). A survey of arthropods in Panama revealed Wolbachia infections in almost $17 \%$ of the species sampled (Werren et al., 1995a), and recent data suggest similar frequencies of Wolbachia infections in nearctic insects as well (Werren \& Windsor, 2000). Furthermore, Wolbachia have been found in mites and filarial nematodes, showing that their distribution extends well beyond insects (Bandi et al., 1998; Breeuwer \& Jacobs, 1996; Sironi et al., 1995). Extrapolation of these estimates of Wolbachia infections suggests that these microbes may be among the most abundant group of parasitic bacteria known, with well over one million species of insects alone infected by Wolbachia (Werren et al., 1995b).

The most commonly described phenotypic effect of Wolbachia is unidirectional cytoplasmic incompatibility (CI), which occurs when an uninfected female mates with an infected male (Breeuwer \& Werren, 1990; Hoffmann \& Turelli, 1997; Hoffmann et al., 1986). Such matings generally 
produce few or no progeny as the result of abortive karyogamy (Callaini et al., 1997; Lassy \& Karr, 1996). Because the other possible types of matings yield normal progeny numbers, the net effect of $\mathrm{Cl}$ is to reduce offspring production of uninfected females compared to infected females. This reproductive advantage to infected females results in the spread of Wolbachia through a population (Caspari \& Watson, 1959; Fine, 1978; Turelli, 1994; Turelli \& Hoffmann, 1991). As Wolbachia spreads, other maternally inherited genomes found in infected females, including mitochondria, are expected to spread with it (Caspari \& Watson, 1959). However, empirical tests of these theoretical predictions of the spread of Wolbachia and associated mitochondrial genomes are currently limited to examples involving Drosophila (Hoffmann et al., 1994; Hoffmann \& Turelli, 1997; Shoemaker et al., 1999; Solignac et al., 1994; Turelli \& Hoffmann, 1991, 1995). This is somewhat surprising, given that the occurrence of Wolbachia sweeps through natural populations is a crucial argument in favour of using these microbes in biological control programmes (Beard et al., 1993; Sinkins et al., 1997).

The population dynamics of Wolbachia in Hymenoptera (such as fire ants) are additionally complicated because uninfected females that mate with Wolbachia-infected males may produce all-male broods rather than show complete reproductive failure (because of the male-haploid genetic system characteristic of the order), or lethality of embyros (Vavre et al., 2000). Furthermore, Wolbachia may induce thelytokous parthenogenesis rather than $\mathrm{Cl}$ in Hymenoptera (Stouthamer, 1997; Stouthamer et al., 1993). Clearly, therefore, additional population studies of Wolbachia are needed, especially in Hymenoptera, in order to better understand the factors that affect their population dynamics within hosts, the effects of infections on the host population genetics, and the potential use of these microbes in biological control of pest populations.

We present the results of a survey for Wolbachia in both native and introduced populations of the fire ant Solenopsis invicta, a serious introduced pest in the USA, as well as in several closely related fire ant species in the $S$. saevissima and $S$. geminata species complexes. Our survey data reveal the presence of variable Wolbachia infections in native (South American) populations of $S$. invicta and S. richteri, but the complete absence of infections in introduced populations of these two species and their hybrids.

\section{Results}

\section{Distribution of Wolbachia in fire ants}

Wolbachia infections were found in six of the nine species surveyed from the $S$. saevissima complex in their native ranges in South America (Table 1). In four of these species, the infections occur at frequencies that are intermediate between zero (complete absence) and one (universal infec- tion). We cannot rule out the possibility that Wolbachia infection also occurs at intermediate frequencies in S. megergates, because of the small sample size for this species. By the same logic, it is possible that the three 'uninfected' species (S. interrupta, S. quinquecuspis and S. electra) do carry Wolbachia at some frequency. In contrast to the widespread occurrence of Wolbachia in South American fire ants, none of the ants screened from the USA appeared to harbour Wolbachia (Table 1). This is true for populations of $S$. invicta and $S$. richteri, and their hybrids, that have been introduced into this country, as well as for the native species S. geminata, which is the member of a species complex which is different from the other ants in this study.

Different populations of native South American S. invicta vary dramatically in their infection status (Table 1). Wolbachia infections were found at intermediate frequencies in both the monogyne $(\mathrm{M})$ and polygyne $(\mathrm{P})$ social forms [The social forms of $S$. invicta and other fire ant species differ in many important reproductive traits (most notably, the number of reproductive queens per colony), and female-mediated gene flow between them is thought to be limited (Ross and Shoemaker 1993; Ross and Shoemaker 1997; Shoemaker and Ross 1996).] sampled from Corrientes, Argentina, but were present at low frequency $(6 \%)$ or were absent in the $\mathrm{M}$ and $\mathrm{P}$ social forms, respectively, from Formosa, Argentina. Frequencies of Wolbachia infections differ significantly between the two regions in Argentina from which native $S$. invicta were sampled (Corrientes and Formosa), as well as between the two social forms within the Corrientes collection locality. However, Wolbachia infection frequencies do not differ significantly between the two social forms of $S$. invicta in Formosa or between the two social forms of S. richteri in Santa Fe, Argentina.

\section{Distribution of Wolbachia in different life stages and body regions}

PCR analyses revealed that Wolbachia are distributed among all life stages and castes of $S$. invicta hosts that were examined, and within all three body regions of the adult queens (Fig. 1). These results are somewhat surprising for workers, given that the ovaries are vestigial in this caste of fire ants (Goetsch, 1953). However, our conclusion that Wolbachia are not confined to adult reproductive tissues is consistent with Dobson et al. (1999), who showed that in some hosts, Wolbachia are found in many tissues throughout the body.

\section{Phylogeny of Wolbachia in S. invicta and S. richteri}

Results of our phylogenetic analyses of the Wolbachia strains based on a portion of the wsp gene, a highly variable gene encoding the bacterial surface protein (Braig et al., 1998; Zhou et al., 1998), are shown in Fig. 2. These phylogenetic analyses are based on wsp sequences from $S$. invicta (sixteen individuals) and $S$. richteri (three individuals), as 
Table 1. Frequencies of Wolbachia infections in native and introduced populations of fire ants. 'Population' column indicates the province or state from which samples were obtained

\begin{tabular}{|c|c|c|c|c|c|c|}
\hline Species & Population & $\begin{array}{l}\text { Native or } \\
\text { introduced }\end{array}$ & $\begin{array}{l}\text { Social } \\
\text { form* }\end{array}$ & $\begin{array}{l}\text { Frequency of } \\
\text { Wolbachia }\end{array}$ & $95 \% \mathrm{Cl}$ & $n \dagger$ \\
\hline S. invicta & Georgia, USA & introduced & M & 0.0 & $0.0-0.09$ & 34 \\
\hline S. invicta & Georgia, USA & introduced & $\mathrm{P}$ & 0.0 & $0.0-0.10$ & 31 \\
\hline S. invicta & Mississippi, USA & introduced & ? & 0.0 & $0.0-0.14$ & 20 \\
\hline S. invicta & Corrientes, Arg. & native & M & 0.86 & $0.75-0.97$ & 36 \\
\hline S. invicta & Corrientes, Arg. & native & $\mathrm{P}$ & 0.56 & $0.42-0.70$ & 43 \\
\hline S. invicta & Formosa, Arg. & native & M & 0.06 & $0.0-0.15$ & 34 \\
\hline S. invicta & Formosa, Arg. & native & $\mathrm{P}$ & 0.0 & $0.0-0.09$ & 34 \\
\hline S. invicta & Santa Fe, Arg. & native & $?$ & 0.0 & & 4 \\
\hline S. invicta & Chaco, Arg. & native & ? & 0.0 & & 3 \\
\hline S. invicta & Santiago del Estero, Arg. & native & $?$ & 0.0 & & 1 \\
\hline S. invicta & Mato Grosso, Brazil & native & ? & 0.0 & & 2 \\
\hline S. invicta & Paraná, Brazil & native & $?$ & 0.0 & & 1 \\
\hline S. richteri & Mississippi, USA & introduced & $?$ & 0.0 & $0.0-0.15$ & 19 \\
\hline S. richteri & Santa Fe, Arg. & native & M & 0.27 & $0.07-0.53$ & 15 \\
\hline S. richteri & Santa Fe, Arg. & native & $\mathrm{P}$ & 0.33 & $0.13-0.60$ & 15 \\
\hline S. invicta/richteri hybrids $\ddagger$ & Mississippi, USA & introduced & $?$ & 0.0 & $0.0-0.18$ & 15 \\
\hline S. macdonaghi & Corrientes, Arg. & native & ? & 0.0 & & 2 \\
\hline S. interrupta & Santiago del Estero and Córdoba, Arg. & native & $?$ & 0.0 & & 5 \\
\hline S. quinquecuspis & Santa Fe and Buenos Aires, Arg. & native & $?$ & 1.0 & & 7 \\
\hline S. saevissima & Minas Gerais, Brazil & native & $?$ & 0.5 & & 2 \\
\hline S. saevissima & Goais, Brazil & native & ? & 1.0 & & 1 \\
\hline S. megergates & Paraná, Brazil & native & ? & 1.0 & & 2 \\
\hline S. Species ' $X$ ' & Santa Fe and Buenos Aires, Arg. & native & $?$ & 0.75 & & 4 \\
\hline S. electra & Santiago del Estero, Arg. & native & $?$ & 0.0 & & 1 \\
\hline S. geminata & Florida, USA & native & ? & 0.0 & & 3 \\
\hline
\end{tabular}

${ }^{*} \mathrm{M}$, monogyne form; $\mathrm{P}$, polygyne form; ?, form unknown.

†Number of individuals (nests).

$\ddagger$ Solenopsis invicta and S. richteri form a large hybrid zone in the USA (see text).

Figure 1. Representative gel of wsp PCR products showing distribution of Wolbachia throughout the body of an adult fire ant. Bands migrating at $400 \mathrm{bp}$ represent the PCR product of the nuclear gene EF- $1 \alpha$, and bands migrating at $600 \mathrm{bp}$ represent the $\mathrm{PCR}$ product of $w s p$. (A) 100 bp DNA size ladder; (B-C) PCR assays of genomic DNA isolated from a single fire ant lacking Wolbachia infection (positive nuclear DNA controls); (D-F) PCR assays of genomic DNA isolated from head, thorax, and gaster (= abdomen), respectively, of a single infected virgin queen fire ant (note that both bands are present and strong using all three body parts as DNA sources); $(G, H)$ negative controls (no genomic DNA).

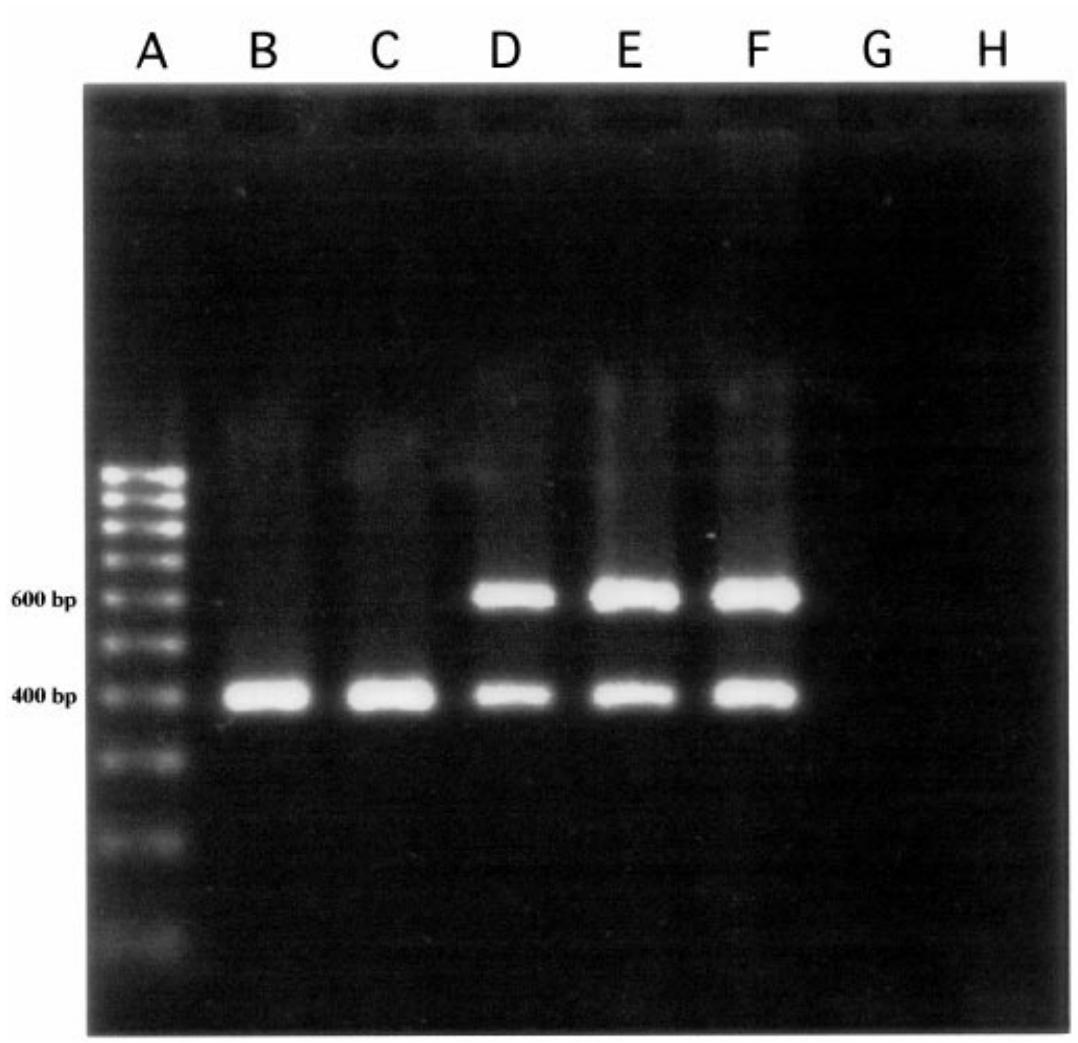




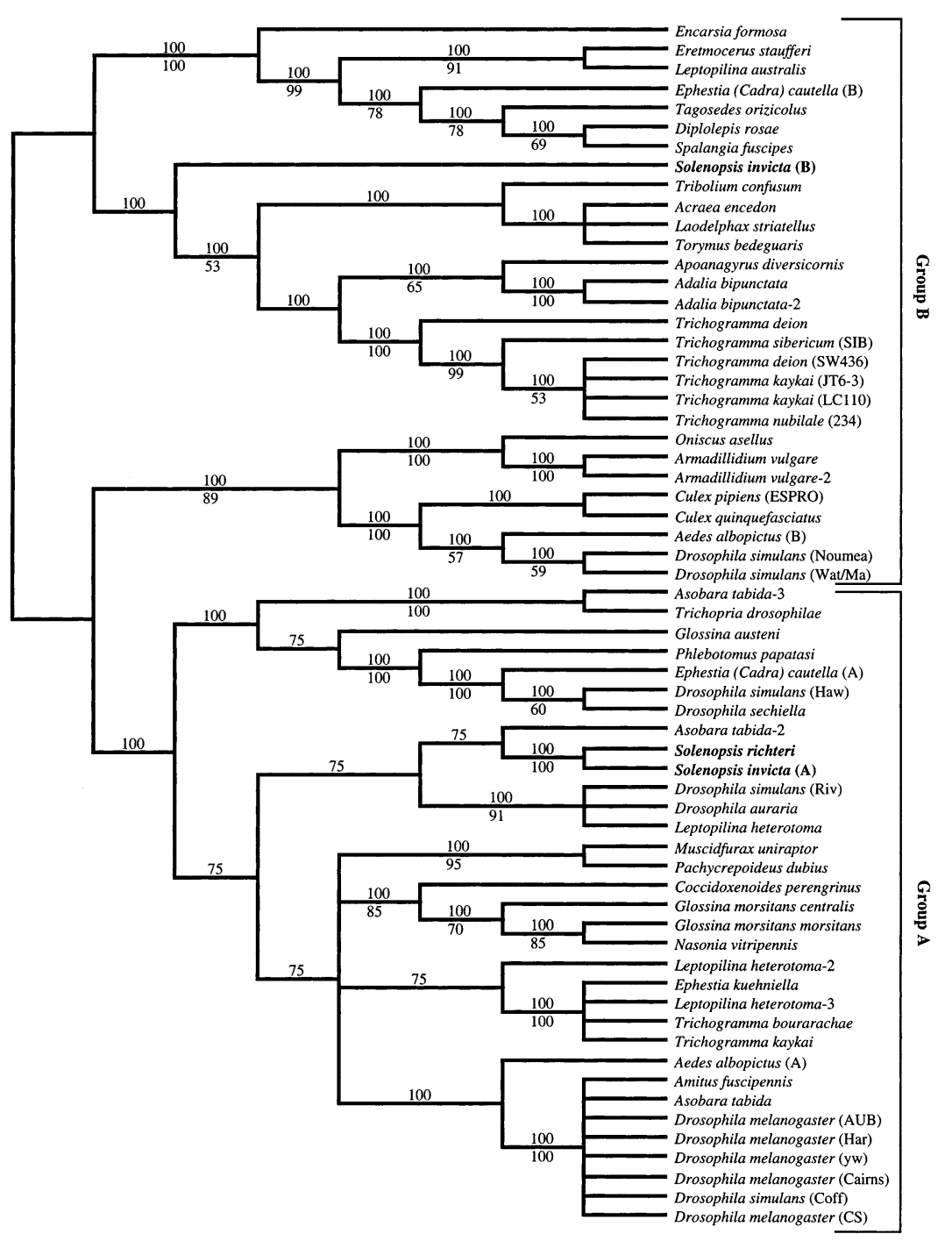

Figure 2. Majority rule consensus parsimony tree (midpoint rooted) for Wolbachia strains based on sequences from the wsp gene. Wolbachia strains are identified by host species from which they were isolated. Wolbachia strains from S. invicta and S. richteri are indicated in bold. Wolbachia strain 'A' was found in six $S$. invicta individuals and three $S$. richteri individuals and strain ' $\mathrm{B}$ ' was found in ten individuals of $S$. invicta. Consistency indices are shown above branches and bootstrap support values (500 replicates) below branches for each node. Bootstrap support values less than fifty are not indicated. well as sequences from fifty-nine other Wolbachia strains from various hosts retrieved from GENBANK. A total of sixtyfour most parsimonious trees were found, with the tree in Fig. 2 representing the $50 \%$ majority rule consensus tree. Differences among the sixty-four most parsimonious trees mainly were confined to placement of taxa near the tips of the tree and to one large unresolved node (polytomy) within the group ' $A$ ' Wolbachia. The neighbourjoining tree (not shown) exhibited a topology very similar to the consensus parsimony tree. The topologies of our trees generally were consistent with those from previous studies (Vavre et al., 1999a; Werren et al., 1995b; Zhou et al., 1998).

We identified two distinct Wolbachia strains within S. invicta. One of the Wolbachia strains from $S$. invicta clustered with strains representing the $A$ group, while the other fell within the $B$ group. These data clearly indicate that two independent infections occurred within this species. Interestingly, all sixteen individuals of $S$. invicta examined carried only a single Wolbachia strain; that is, none had double infections, even though both A and B Wolbachia are found within both social forms in Corrientes. Furthermore, all of the wsp sequences from each Wolbachia group present in $S$. invicta were identical (six group A and ten group B sequences). Only one Wolbachia strain was identified in each of the three individuals of $S$. richteri examined, and the three wsp sequences were identical. Finally, our sequence data reveal that the Wolbachia A strain from S. invicta and the strain from $S$. richteri were nearly identical, differing by only a single synonymous substitution.

\section{MtDNA phylogenetic analyses}

MtDNA phylogenetic analyses were based on sequences from the COI and COIl genes in a subset of the individuals 
Figure 3. Majority rule consensus parsimony tree for mtDNA haplotypes based on sequences from the cytochrome oxidase I (COI) and cytochrome oxidase II (COII) genes. Consistency indices are shown above branches and bootstrap support values ( 500 replicates) below branches for each node. Bootstrap support values less than fifty are not indicated.

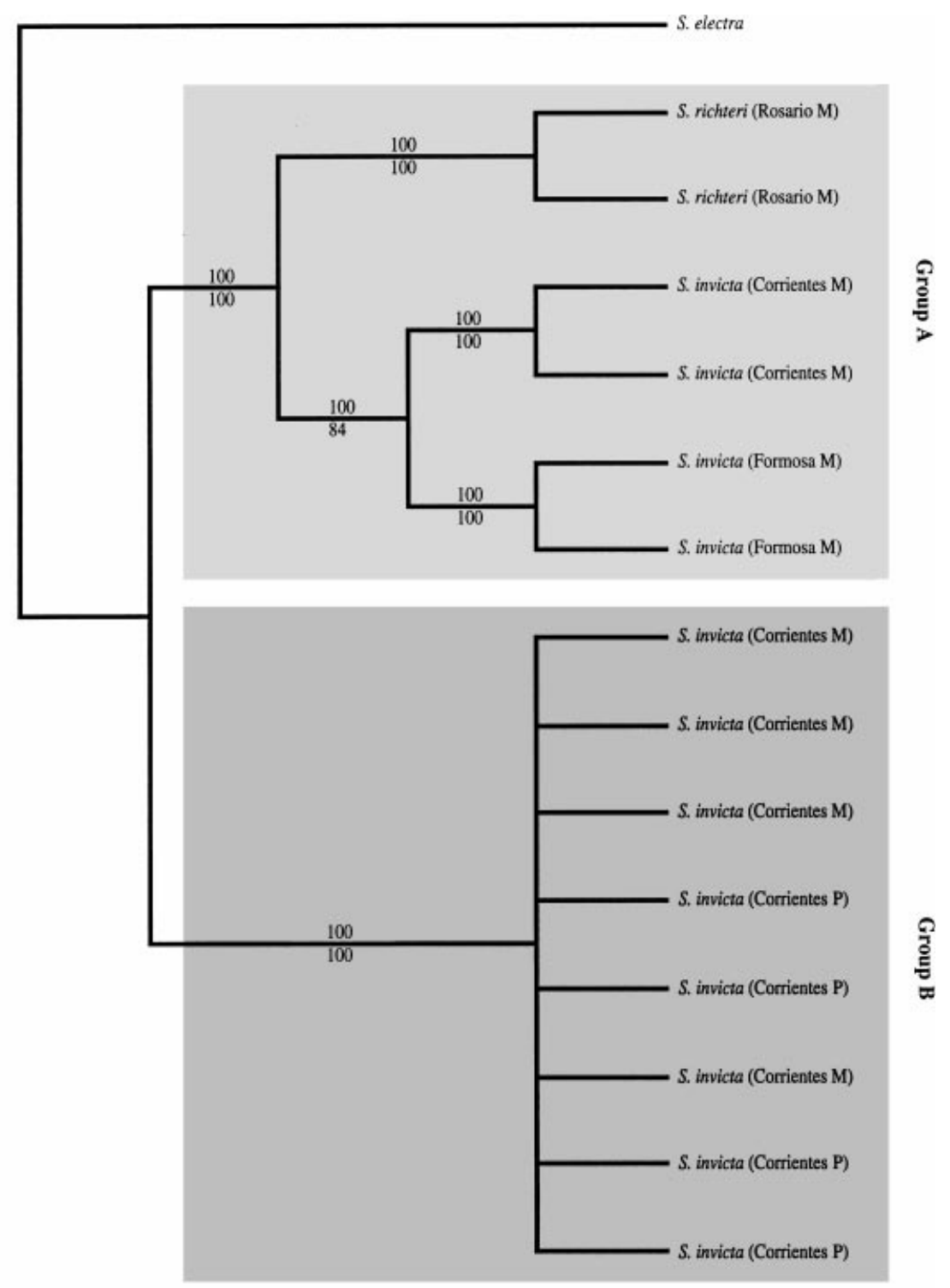

above (twelve $S$. invicta and two $S$. richteri), as well as from an individual of $S$. electra, which served as the outgroup taxon. A total of four most parsimonious trees were found, which differed only in the relationships of $S$. invicta haplotypes within one clade (Fig. 3). The neighbourjoining tree featured a virtually identical topology. Our results show that mtDNA haplotypes from $S$. invicta are paraphyletic, with one group of mtDNA haplotypes sharing a more recent ancestor with haplotypes from $S$. richteri than with other $S$. invicta haplotypes. This paraphyletic relationship was resolved with a high bootstrap support value in all four parsimony trees, as well as in the neighbourjoining tree.

Significantly, there was complete concordance between the particular Wolbachia strain carried by an individual and the haplotype clade in which its mtDNA was placed (Fig. 3). That is, the mtDNA from all individuals carrying Wolbachia strain $B$ formed a monophyletic group that was distinct from the mtDNA of individuals carrying strain $A$, with the latter individuals representing both $S$. invicta and $S$. richteri. Thus, the paraphyly of mtDNA haplotypes in $S$. invicta apparently can be explained by some force that affects the entire cytoplasm rather than just this particular organellar genome.

\section{Discussion}

Wolbachia are currently of interest to a broad spectrum of biologists because of their widespread distribution, varied phenotypic effects on hosts, potential role in speciation, and 
potential use in biological control programmes (Hoffmann \& Turelli, 1997; Sinkins et al., 1997; Stouthamer, 1997; Werren, 1997, 1998; Werren \& O'Neill, 1997 and references therein). However, detailed studies examining the frequencies of Wolbachia infections within and among natural populations are rare, and mostly are limited to Drosophila (Ballard et al., 1996; Hoffmann, 1988; Hoffmann et al., 1994, 1996, 1998, 1990; Shoemaker et al., 1999; Turelli \& Hoffmann, 1991, 1995). This is surprising given the widespread interest in these microbes, the substantial amount of theory developed on their population dynamics, and the crucial need for such data in assessing the potential for using Wolbachia in biological control programmes (Sinkins et al., 1997; Turelli, 1994; Turelli \& Hoffmann, 1999; Werren, 1997, 1998; Werren \& O'Neill, 1997). In the present paper, we present the results of a survey for Wolbachia in native and introduced populations of the fire ant $S$. invicta, as well as in several other fire ant species. Our study represents the first survey of the frequencies of Wolbachia infections within natural populations of a social hymenopteran insect of significant economic importance.

\section{Patterns of Wolbachia infection in native and introduced fire ants}

Our survey data revealed that individuals from native populations of both $S$. invicta and $S$. richteri often harbour Wolbachia infections, whereas individuals representing both species from the USA, where they have been introduced, apparently lack Wolbachia (Table 1). Several possible explanations exist for these distinct patterns, including: (i) the absence of Wolbachia in all of the original foundresses introduced into the USA, (ii) the loss of Wolbachia infections in introduced fire ants due to drift or selection, (iii) the failure to detect Wolbachia in introduced ants (due to low frequency of infection) even though it is present, and (iv) the introduction and spread of Wolbachia in native $S$. invicta and $S$. richteri populations after introduction of these species into the USA. We briefly address each of these possibilities in turn.

The absence of Wolbachia in introduced fire ants may stem from the absence of these microbes in all of the original foundresses introduced into the USA. Previous genetic data clearly show that $S$. invicta experienced a severe population bottleneck associated with its introduction (Ross \& Trager, 1990; Ross et al., 1993, 1996b), and the same is almost assuredly true for $S$. richteri. The inferred small number of foundresses introduced into the USA, coupled with the fact that many colonies of both $S$. invicta and S. richteri in South America lack Wolbachia (i.e. Wolbachia infections frequencies are variable within and between populations), suggests that this possibility is not unlikely. $A$ related scenario holds that Wolbachia infections are absent in the USA because the original foundresses are derived from a native population that completely lacked
Wolbachia. This is also not an unlikely scenario for $S$. invicta, especially given the fact that we did not detect Wolbachia infections in the three $S$. invicta colonies sampled from Brazil, the suspected source country of introduced ants of this species (Table 1).

It is also possible that some proportion of the original foundresses carried Wolbachia, but that the infections were subsequently lost due to drift and/or selection. Recent theory has shown that there is a threshold frequency that must be obtained for these microbes to increase in frequency and sweep to fixation or near-fixation (which varies depending on the fitness effects and maternal transmission rate), and that the equilibrium frequency is zero (i.e. loss of the bacteria) below this threshold (Turelli, 1994). Thus, if relatively few foundresses carried Wolbachia, the particular female lineages carrying these microbes may have been lost either through drift or selection associated with the fitness costs of carrying the bacteria. Consistent with this possibility, we find that one of the common mitochondrial haplotypes associated with Wolbachia in South America is common among fire ants in the USA (Shoemaker and Ross, unpublished data).

We consider it unlikely that Wolbachia are present in introduced fire ants but that we failed to detect them, given our large sample sizes (119 individuals of S. invicta, $S$. richteri, and their hybrids). Indeed, to go undetected, the frequencies of Wolbachia infections in the USA would have to be so low that the predicted stable equilibrium would likely be complete loss of the bacteria through drift or selection (Turelli, 1994).

We also consider it unlikely that the lack of Wolbachia in introduced fire ants occurs because Wolbachia invaded native populations after the introduction of fire ants into the USA, given the levels of mtDNA variation within the two groups of native $S$. invicta infected by Wolbachia (A and $B$ in Fig. 3). Because Wolbachia are maternally transmitted, as Wolbachia spreads through a population all other maternally inherited organelles, including mitochondria, 'hitchhike' along with it (Turelli, 1994; Turelli \& Hoffmann, 1991, 1995). Thus, a Wolbachia-associated sweep of a given mtDNA haplotype eliminates all variation, and any sequence variation detected among the extant mtDNA haplotypes represents mutations that have arisen since the Wolbachia sweep (Caspari \& Watson, 1959; Fine, 1978; Turelli, 1994; Turelli \& Hoffmann, 1991). Given that $S$. invicta was introduced in the early twentieth century, the expected amount of mtDNA variation within these two groups is essentially zero (assuming a $2 \%$ sequence divergence of mtDNA per million years; Brower, 1994; Guillemaud et al., 1997; Venanzetti et al., 1993). The presence of any mtDNA variation among infected native ants would be surprising under this scenario.

Our data on mtDNA variation suggest that Wolbachia have been present in $S$. invicta and $S$. richteri for a very 
long period. We found several mtDNA haplotypes within each group of Wolbachia in S. invicta (see below) and, at least in the case of the four haplotypes sequenced from $S$. invicta group A, relatively high between-haplotype divergence. Estimates of $\pi$, the average number of nucleotide differences per site, for haplotypes from groups $A$ and $B$ were $0.01795(n=4)$ and $0.00083(n=8)$, respectively. These estimates, although based on a small number of sequences, suggest that the two Wolbachia strains have been present within $S$. invicta for at least 40000 years (and much longer than that in the case of group A; Brower, 1994; Guillemaud et al., 1997; Venanzetti et al., 1993). Thus, Wolbachia were most likely present in native $S$. invicta and $S$. richteri populations long before the introduction of these two species into the USA.

Our survey also revealed that Wolbachia infection frequencies differed significantly between the two geographical populations of native $S$. invicta (Formosa and Corrientes, Argentina), as well as between the two social forms in the Corrientes population. These data are concordant with previous results showing significant differentiation between the two regions and between the two social forms within a single region for both mitochondrial and nuclear DNA markers, indicating reduced levels of gene flow (Ross et al., 1997). Such concordant patterns of genetic structure recorded from the Wolbachia and mtDNA genomes are expected if the two genomes are inherited in a similar fashion, that is, only through females (no paternal leakage) and with no horizontal transfer (Caspari \& Watson, 1959; Fine, 1978; Turelli, 1994).

The differences in Wolbachia infection frequencies that we detected between the two social forms may also stem from differences in the selective regimes acting on this microbe in each form. In this respect, the predicted effects of $\mathrm{Cl}$ and parthenogenesis-inducing (PI) Wolbachia in $S$. invicta (production of males instead of females) parallel another interesting phenomenon observed in $S$. invicta that is associated with the genetic sex-determination system. Sex in fire ants, as in many other social Hymenoptera, is presumably determined by genotype at a single nuclear locus, with individuals heterozygous at this locus developing into females and individuals hemizygous or homozygous developing into males (Crozier, 1971; Ross \& Fletcher, 1985, 1986; Ross et al., 1993). When a female mates with a male carrying an allele identical to one of hers at the sex locus, $50 \%$ of her diploid offspring will be homozygous at this locus and develop into diploid males (Crozier, 1971; Ross \& Fletcher, 1985; Ross et al., 1993). Such diploid-maleproducing queens occur at a frequency of around $20 \%$ in newly mated polygyne (P) queens and monogyne (M) queens of $S$. invicta in the USA; however, such queens are never found heading mature $\mathrm{M}$ colonies, because the colonies they found invariably fail (Ross \& Fletcher, 1985, 1986; Ross \& Shoemaker, 1997; Ross et al., 1993). This differential mortality is caused by diploid-male-producing queens investing their limited resources in diploid males rather than in the workers that are crucial to early colony survival. Polygyne queens are not subject to this source of mortality because they do not attempt to found colonies independently but instead enter established nests to become egg layers (Ross \& Fletcher, 1985, 1986; Ross \& Shoemaker, 1997). Although we currently do not know what effects these microbes have on fire ants (see below), we predict a similar pattern of selection against uninfected $\mathrm{M}$ queens carrying $\mathrm{Cl}$ or PI Wolbachia, because such queens would presumably produce only haploid males or diploid males, respectively, at the crucial colony-founding stage.

The predicted outcome of such strong selection in $\mathrm{M}$ queens compared to $\mathrm{P}$ queens is a faster sweep and higher equilibrium frequency of $\mathrm{Cl}$ Wolbachia infections in the $\mathrm{M}$ form than the $P$ form. This conclusion follows from the findings that the dynamics and equilibrium frequency of Wolbachia infections are mostly governed by the parameters outlined by Turelli (1994), namely: (i) $F$, the fecundity of infected relative to uninfected females (ii) $H$, the hatch rate of incompatible relative to compatible fertilizations, and (iii) $\mu$, the fraction of uninfected ova produced by infected females (i.e. maternal transmission fidelity). The differences in social biology between the two forms discussed above imply that the reproductive advantage to infected females will be greater in the $\mathrm{M}$ social form (Caspari \& Watson, 1959; Fine, 1978; Turelli, 1994). If maternal transmission of Wolbachia is incomplete (as suggested by preliminary laboratory studies; Shoemaker et al. unpublished results), then these $\mathrm{P}$ queens may contribute some uninfected females, as well as males, to the population every generation. The effects will be a slower sweep of Wolbachia in the $\mathrm{P}$ form (because $H$, the hatch rate of incompatible relative to compatible fertilizations, will be greater in uninfected queens of the $P$ form) as well as a lower equilibrium frequency of Wolbachia infections in the $\mathrm{P}$ form. This latter prediction is consistent with our survey data showing higher infection frequencies in the $\mathrm{M}$ form. Additionally, the persistence of incompatibly mated queens could impose a continuous fitness cost on polygyne colonies due to the production of male progeny (or lethality of incompatible eggs). Modelling is needed to determine more exactly the population dynamics of Wolbachia in monogyne and polygyne fire ant populations.

\section{MtDNA and Wolbachia variation}

There are three explanations for the paraphyly of $S$. invicta mtDNA haplotypes, namely: (i) individuals from the two mtDNA clades represent two species that are morphologically indistinguishable (cryptic species), (ii) there has been historical introgression between $S$. invicta and S. richteri, resulting in capture of cytoplasmic genomes, and (iii) S. richteri 
originated from within the $S$. invicta clade. Allozyme data generally support the morphology-based identification of S. invicta individuals in this study (Ross et al., 1997; Ross \& Trager, 1990; Ross et al., 1996b); however, the allozyme data also reveal modest but statistically significant differentiation between the $S$. invicta of the two mtDNA clades in Corrientes (Shoemaker et al. unpublished data). This finding is consistent with the hypothesis that the ants identified as $S$. invicta from Corrientes actually represent cryptic species, and that these have been reproductively isolated for a sufficient period for significant nuclear genetic divergence to evolve. That sufficient time may have elapsed to allow such nuclear differentiation is suggested by the $m t D N A$ divergence seen in these two groups of $S$. invicta. Total divergence estimates of $\pi$, the average number of nucleotide differences per site, for all $S$. invicta haplotypes that we sampled is 0.027 , suggesting a time to common ancestry greater than one million years. Furthermore, previous estimates of $d$, the average pair-wise sequence divergence, for RFLP mtDNA haplotypes from a larger set of individuals from Corrientes are 0.034 and 0.041 (for the $\mathrm{M}$ and $\mathrm{P}$ forms, respectively). Future analyses involving additional nuclear DNA markers are necessary to resolve the issue of possible cryptic species within nominal $S$. invicta in South America.

The paraphyly of $S$. invicta $\mathrm{mtDNA}$ also could be explained by a hybridization event between $S$. invicta and $S$. richteri that resulted in capture of the cytoplasmic genome of $S$. richteri by $S$. invicta. Previous studies have shown that introduced populations of these two species form a large hybrid zone where they come into contact (Ross et al., 1987; Shoemaker et al., 1994). Although extensive hybridization apparently does not occur at present in South America, allozyme studies suggested at least some level of hybridization (Ross \& Trager, 1990), and even very limited amounts of historical interspecific gene flow may be sufficient for complete capture of the cytoplasmic genomes when Wolbachia are involved. Indeed, even a single hybridization event followed by subsequent backcrossing to one species may introduce Wolbachia into a population previously lacking it. If infection frequencies subsequently drift high enough, then the selective advantage of carrying Wolbachia (i.e. causing $\mathrm{Cl}$ ) will be realized and the microbe will increase in frequency in the population (Hoffmann \& Turelli, 1997; Turelli, 1994; Turelli \& Hoffmann, 1995), carrying with it the 'foreign' mtDNA. The end result of such a selective sweep will be the capture of the cytoplasmic genomes of one species by another, with essentially no signal of hybridization in the nuclear genome (Rieseberg et al., 1991).

Finally, as mentioned before with respect to infection frequencies, the complete concordance of the mitochondrial and Wolbachia genomes in S. invicta suggests that the inheritance patterns of these genomes are similar (i.e. maternal inheritance), and that significant amounts of horizontal transfer or paternal leakage of Wolbachia do not occur.

\section{Phenotypic effects of Wolbachia on fire ants}

Possible phenotypic effects of Wolbachia on fire ants, some of which have been described recently in other arthropods, include: (i) cytoplasmic incompatibility $(\mathrm{Cl})$, (ii) parthenogenesis induction (PI) (Stouthamer et al., 1993), (iii) mutualistic interactions with the host, perhaps increasing host fitness (Vavre et al., 1999b), (iv) pathogenic relationships with the host (i.e. systemic infections; (Min \& Benzer, 1998)), (v) no $\mathrm{Cl}$ but the ability to rescue females from $\mathrm{Cl}$ caused by other Wolbachia strains (Bourtzis et al., 1998; Merçot \& Poinsot, 1998), (vi) male killing (Hurst et al., 1999), or (vii) lack of any apparent effects, with the microbe behaving as a neutral cytoplasmic element (Clancy \& Hoffman, 1996).

Choosing among these possibilities is speculative at present, but two points are worthy of mention. First, parthenogenesis induction can be ruled out, at least by the mechanism previously described (Stouthamer et al., 1993). Parthenogenesis in infected hymenopteran females carrying PI Wolbachia is caused by the process of gamete duplication, which typically results in a diploid female that is homozygous at every locus. However, in the case of fire ants, gamete duplication would result in a diploid male rather than a diploid female because of homozygosity at the sex-determination locus. We consistently observed Wolbachia-infected female progeny in our analyses, a finding inconsistent with gamete duplication. Second, inference of the phenotypic effects of Wolbachia is not aided by our phylogenetic analyses. This is because one Wolbachia strain from fire ants $(B)$ is part of a clade containing strains that have diverse host effects $(\mathrm{Cl}, \mathrm{PI}$, male killing), while the other $(A)$ is not similar enough to previously described strains to infer its effects. It should be noted that the coexistence of different Wolbachia strains, such as those we found in $S$. invicta, is not predicted within panmictic host populations (Caspari \& Watson, 1959; Turelli, 1994; but see Rousset et al. (1991) for a similar example in mosquitoes). One possible explanation for their coexistence is that the two strains have different phenotypic effects; for instance, one may induce $\mathrm{Cl}$ while the other induces male-killing.

\section{Biological control implications}

Wolbachia may have significant fitness effects on fire ants, either directly or due to cytoplasmic incompatibility. The occurrence of Wolbachia at intermediate frequencies would impose a $\mathrm{Cl}$-induced fitness cost on populations due to incompatibilities between infected and uninfected individuals. Thus, these bacteria could reduce fire ant populations in North America, a noteworthy consideration given the significant pest status of fire ants and the desire to find new natural control agents (Lofgren, 1986; Lofgren et al., 1975; Patterson, 1994). The diminished genetic load on 
the introduced pest populations, which are free of infection, may help explain the five- to tenfold increase in colony densities of this ant in the USA compared to its native range (Porter et al., 1992). Furthermore, a finding of any significant load on fire ants caused by Wolbachia (direct fitness effects of Wolbachia infection, $\mathrm{Cl}$ coupled with imperfect transmission, or some other effect) would mean that there is considerable potential for successfully incorporating these microbes into an integrated pest management programme to suppress populations of introduced fire ants. Therefore, determination of the effects of Wolbachia on $S$. invicta is an important topic for future research.

\section{Experimental procedures}

\section{Collection and identification of ants}

Collection localities for all ants surveyed in the present study are shown in Table 1. Introduced fire ants representing S. invicta and S. richteri were collected in the spring of 1990 and fall of 1995. The majority of such S. invicta samples were from Monroe, Georgia, USA (see Ross and Shoemaker, 1997, Ross et al., 1999 and Shoemaker and Ross, 1996 for descriptions of this locality). A total of sixty-five colonies at this locality was used for the present study; thirty-four of these colonies were of the monogyne (M) social form and thirty-one were of the polygyne $(P)$ form. Additional colonies of $S$. invicta of unidentified social form were collected from Vaiden, Mississippi, USA (four colonies), Morgan City, Mississippi (eight), and Durant, Mississippi (eight). Solenopsis richteri nests of unidentified social form were collected from Nixon, Mississippi (nine), Randolph, Mississippi (nine), and Banner, Mississippi (one). Finally, hybrid S. invicta/richteri colonies of unidentified social form were sampled from Greenwood, Mississippi (six), Coffeeville, Mississippi (seven), Holcomb, Mississippi (one), and Yalobusha County, Mississippi (one). All of the above colonies previously have been confirmed as nests of either $S$. invicta, $S$. richteri or hybrids using at least three diagnostic allozyme markers (Shoemaker et al., 1996).

Native fire ants representing $S$. invicta of both social forms were collected in 1992 from two populations located near the cities of Corrientes and Formosa in north-Eastern Argentina. Over thirty colonies of each social form in each population were sampled (Table 1). These populations, which are separated by the Río Paraná $(\approx 160 \mathrm{~km}$ ), have been the subject of extensive previous genetic studies (Ross, 1997; Ross et al., 1993, 1996a,b, 1997), so we are confident that the classification of nests to both species and social form is correct (Ross et al., 1993). Ants from eleven additional colonies of $S$. invicta (unknown social form) were collected in the fall of 1988 and 1998 from the following locations: Santa Fe, Argentina (four colonies); Santiago del Estero, Argentina (one); Chaco, Argentina (three); Mato Grosso, Brazil (two) and Paraná, Brazil (one). Samples of native S. richteri of both social forms (fifteen colonies of each) were collected in 1992 from a population located near the city of Rosario in central Argentina. Social form of each colony was determined by examining the genotypes of twelve nestmate workers at seven polymorphic allozyme loci; genotype distributions inconsistent with the workers being full sisters are diagnostic of $P$ colonies (Ross, 1992; Ross \& Shoemaker, 1993; Ross et al., 1988, 1999).
Samples of seven additional species of fire ants in the S. saevissima species complex were collected from their native South American ranges in 1988, 1992 and 1998 (one to seven nests per species). Species identifications were initially made in the field using a published morphological key and were subsequently confirmed in the laboratory using both morphological characters and allozyme markers that exhibit species-specific allele compositions (Ross et al., 1993, 1997; Ross \& Trager, 1990; Trager, 1991). Any nests for which species identifications disagreed between these two character sets were excluded, except for samples of Species ' $\mathrm{X}$ ', a morphologically cryptic species that is distinguished from all the other species by the possession of unique alleles at two allozyme loci (Ross \& Trager, 1990). The social form of colonies of these additional species is unknown. For further information on the collection localities and methods for assigning nests to species, see Table 1, Ross (1997), and Ross et al. (1993, 1996a,b, 1997).

Finally, three colonies of $S$. geminata of unknown social form were sampled in its native range from Tampa (one colony) and Tallahassee (two), Florida, USA. This fire ant is placed in a different species complex (S. geminata complex) than the other species we studied.

\section{Screening for Wolbachia}

Total genomic DNA was isolated from each individual using the Puregene ${ }^{\circledR}$ DNA isolation kit, a simple method for isolating high molecular weight DNA (Ross \& Shoemaker, 1997). Genomic DNA from each ant was screened for the presence of Wolbachia via the polymerase chain reaction using the primers Wsp81F and Wsp691R (Zhou et al., 1998). The wsp primers amplify a portion of a highly variable gene encoding the bacterial surface protein (Braig et al., 1998; Zhou et al., 1998). Details of the PCRs, PCR profiles, and electrophoresis of products are described in Shoemaker et al. (1999) and Zhou et al. (1998).

Wsp PCRs were performed in $15 \mu \mathrm{l}$ volumes containing $13 \mu \mathrm{l}$ of Platinum® PCR SuperMIX (Gibco BRL), $0.18 \mu \mathrm{l}$ of a $25 \mu \mathrm{M}$ solution of each primer (Wsp81f and Wsp691R), and $1-3 \mu \mathrm{l}$ of genomic DNA. Also included in each reaction were two control primers $(0.12 \mu \mathrm{l}$ of a $25 \mu \mathrm{M}$ solution of each): EF $1 \alpha-532 \mathrm{~F}$ (5'AGGCAAATGTCT TATTGAAG- $3^{\prime}$ ) and EF1 $\alpha-610 R$ ( $5^{\prime}$-GCGGGTGCGAAGGTAACAAC-3'). These primers amplify a 400 bp portion of one of the two copies of the nuclear gene EF1 $\alpha$ (elongation factor). Inclusion of these two primers in every PCR reaction constitutes an important positive control for determining the infection status of individuals. The presence of the EF1 $\alpha$ fragment and absence of the Wolbachia-specific fragment most likely reflects an absence of the bacteria rather than low quality or overly concentrated genomic DNA or an error associated with PCR setup. On the other hand, when such control primers fail to work, one cannot confidently assert that the lack of a Wolbachia-specific PCR product results from an absence of the bacteria. Therefore, in cases where the EF1 $\alpha$ fragments were absent, we serially diluted genomic DNA and performed PCR again.

Amplifications were carried out in a Perkin Elmer 9700 thermocycler programmed as follows: $1 \mathrm{~min}$ at $94{ }^{\circ} \mathrm{C}$ for one cycle; $30 \mathrm{~s}$ at $94^{\circ} \mathrm{C}, 30 \mathrm{~s}$ at $60^{\circ} \mathrm{C}\left(-1_{2}^{\circ}{ }^{\circ} \mathrm{C}\right.$ per cycle $)$ and $1 \mathrm{~min}$ at $72{ }^{\circ} \mathrm{C}$ for 10 cycles (touchdown PCR); $30 \mathrm{~s}$ at $94^{\circ} \mathrm{C}, 30 \mathrm{~s}$ at $53^{\circ} \mathrm{C}$, and $1 \mathrm{~min}$ at $72{ }^{\circ} \mathrm{C}$ for twenty-five cycles; $5 \mathrm{~min}$ at $72^{\circ} \mathrm{C}$ for one terminal cycle. Approximately $5 \mu \mathrm{l}$ of each PCR reaction mixture was electrophoresed in $2 \%$ agarose gels. Gels were stained with ethidium bromide and bands visualized under UV illumination. Images of 
stained gels were photographed and stored electronically using a gel photodocumentation system.

A single individual from each sampled fire ant colony was screened for Wolbachia to estimate population infection frequencies. A bootstrapping procedure was used to estimate the $95 \%$ confidence intervals $(\mathrm{Cls})$ around nonzero frequency estimates for all samples with more than five individuals. Individuals were sampled randomly (with replacement) from the original data set for each bootstrap replicate. This procedure was repeated 1000 times, and the $95 \%$ Cls were found by eliminating the twenty five lowest and twenty five highest values from the ordered array of the 1000 estimates. In cases where infection frequencies were apparently zero and sample sizes were greater than five, 95\% Cls were estimated from the binomial distribution as the interval zero to $f^{*}$, where $f^{*}$ represents the upper limit frequency of infection in the population in which Wolbachia infections may go undetected $95 \%$ or more of the time for a particular sample size. Samples with $\mathrm{Cls}$ that do not overlap are taken to have significantly different infection frequencies.

\section{Distribution of Wolbachia within S. invicta}

We conducted two analyses to determine the distribution of Wolbachia among the different life stages and castes of $S$. invicta, as well as among the different body regions of adult sexuals. We screened for Wolbachia using the PCR assay described above using individuals of the following life stages and castes (two individuals each): fourth instar worker larvae; worker pupae; worker adults; queen pupae; adult virgin queens; male pupae; adult males. All of these individuals were taken from two field-collected monogyne colonies known to have Wolbachia-infected queens. To determine the distribution of Wolbachia throughout the body, we sectioned two adult virgin queens into three parts (head, thorax, gaster [= abdomen]), performed DNA isolations on each part, and screened for Wolbachia using our PCR assay described above.

\section{Sequencing of Wolbachia strains and $m t D N A$}

A portion of the wsp gene $(\approx 590 \mathrm{bp}$ ) was sequenced from a total of nineteen infected individuals (sixteen $S$. invicta and three S. richteri - see Fig. 2) using the primers Wsp 81F and Wsp 691R (Zhou et al., 1998). For sequencing, Wolbachia DNA was PCRamplified as described above, except $50 \mu$ l volumes were used, no control primers were included, and the final extension at $72{ }^{\circ} \mathrm{C}$ was for $45 \mathrm{~min}$ rather than $5 \mathrm{~min}$. PCR amplicons were gel-purified using Qiagen gel extraction spin columns and used directly in standard fluorescent cycle-sequencing PCR reactions (ABI Prism Big Dye terminator chemistry). Sequencing reactions were cleaned using sephadex columns (Princeton Separations) and run on an automated $\mathrm{ABI}$ Prism 310 sequencer.

Sequences were aligned to a subset of previously published Wolbachia sequences from other insects and arthropods (fifty nine sequences found in GenBANK, submitted by Braig et al. 1998; Hurst et al. 1999; Schulenburg et al. 2000; van Meer et al. 1999; Vavre et al. 1999a; Zhou et al. 1998). The sequence data were used to construct phylogenetic trees of Wolbachia strains using maximum parsimony and neighbour-joining methods, as implemented in PAUP* 4.0 (Swofford, 1999). The third, hypervariable region (positions 521-567) was excluded for these analyses (Vavre et al., 1999a; Zhou et al., 1998). Parsimony trees were constructed using the heuristic search option (1000 random additions) of PAUP* 4.0 and the resulting trees were midpoint rooted.
Bootstrap values were generated using the heuristic search algorithm (500 bootstrap replicates with ten random addition searches per replicate). The neighbour-joining tree was constructed using Jukes-Cantor distances, and the resulting tree was midpoint rooted. Wsp sequences representing each Wolbachia strain have been deposited in GenBANK (AF243435-AF243437).

A $910 \mathrm{bp}$ portion of the mitochondrial (mtDNA) genome was sequenced from a subset of the individuals above (twelve $S$. invicta and two $S$. richteri), as well as from an additional individual representing $S$. electra (see Fig. 3). MtDNA PCR amplifications were carried out using the primer C1-J-2195 (COI-RLR; 5'TTGATTTTTTGGTCATCCAGAAGT-3'; see Simon et al., 1994) and a primer we designed (DDS-COII-4; 5'-TAAGATGGTTAATGAAGAGTAG-3'; Ross \& Shoemaker, 1997). These two primers amplify a portion of the mtDNA that includes regions of both the cytochrome oxidase I (COI) and cytochrome oxidase II (COII) genes. MtDNA PCRs were performed in $50 \mu$ l volumes containing $5 \mu \mathrm{l}$ of $10 \times$ buffer (Gibco BRL), $3 \mu \mathrm{l}$ of a $1.5-\mathrm{mm}$ solution of magnesium chloride, $0.6 \mu \mathrm{l}$ of $10 \mathrm{~mm}$ solutions of dNTPs, $1.0 \mu \mathrm{l}$ of $25 \mu \mathrm{m}$ solutions of each primer, 1-3 $\mu \mathrm{l}$ of genomic DNA, and $0.2 \mu \mathrm{l}$ (1 unit) of thermostable (Taq) DNA polymerase (Gibco BRL). Amplifications were carried out in a Perkin-Elmer 9700 thermocycler programmed as follows: $1 \mathrm{~min}$ at $94^{\circ} \mathrm{C}$ for one cycle; $30 \mathrm{~s}$ at $94^{\circ} \mathrm{C}, 1 \mathrm{~min}$ at $50^{\circ} \mathrm{C}$, and $2 \mathrm{~min}$ at $68^{\circ} \mathrm{C}$ for thirty-five cycles; 5 min at $72{ }^{\circ} \mathrm{C}$ for one terminal cycle.

The 910 bp mtDNA amplicons were gel-purified using Qiagen gel extraction spin columns, used in standard fluorescent cyclesequencing PCR reactions (ABI Prism Big Dye terminator chemistry), and run on an automated ABI Prism 377 or 310 sequencer as described above. Sequences were aligned and phylogenetic trees were constructed using maximum parsimony and neighbourjoining as implemented in PAUP* 4.0 (Swofford, 1999). Parsimony trees were constructed using the branch and bound search option, and the resulting trees were rooted using $S$. electra as an outgroup. Previous morphological analyses place this species in a different but closely related species subcomplex than the one to which S. invicta and S. richteri belong (Trager, 1991). Bootstrap values were generated using a heuristic search algorithm (1000 bootstrap replicates with ten random addition searches per replicate). The neighbour-joining tree was constructed using Jukes-Cantor distances and was rooted also using $S$. electra as an outgroup.

\section{Acknowledgements}

We thank Santiago Navarro and Hollie Rebo for laboratory assistance, and Dave Cowan for helpful comments on the manuscript. This research was supported by grants from USDA to DDS (1999-05107) and to K.G.R., D.D.S. \& J.V. McHugh (1999-02465), from the NSF to K.G.R. (9707331), and from the National Geographic Society to K.G.R. (6127-98).

\section{References}

Ballard, J.W.O., Hatzidakis, O.J., Karr, T.L. and Kreitman, M. (1996) Reduced variation in Drosophila simulans mtDNA. Genetics 144: 1519-1528.

Bandi, C., Anderson, T.J.C., Genchi, C. and Blaxter, M.L. (1998) Phylogeny of Wolbachia in filarial nematodes. Proc $R$ Soc Lond B 265: 2407-2413. 
Beard, C.B., O'Neill, S.L., Tesh, R.B., Richards, F.F. and Aksoy, S. (1993) Modification of arthropod vector competence via symbiotic bacteria. Parasitol Today 9: 179-183.

Bourtzis, K., Dobson, S.L., Braig, H.R. and O'Neill, S.L. (1998) Rescuing Wolbachia have been overlooked ... Nature 391 : 852-853.

Braig, H.R., Zhou, W., Dobson, S. and O'Neill, S.L. (1998) Cloning and characterization of a gene encoding the major surface protein of the bacterial endosymbiont Wolbachia. J Bacteriol 180: 2373-2378.

Breeuwer, J.A.J. and Jacobs, G. (1996) Wolbachia: Intracellular manipulators of mite reproduction. Exp Appl Acarol 20: 421434.

Breeuwer, J.A.J., Stouthamer, R., Barns, D.A., Pelletier, D.A., Weisburg, W.G. and Werren, J.H. (1992) Phylogeny of cytoplasmic incompatibility microorganisms in the parasitoid wasp genus Nasonia (Hymenoptera: Pteromalidae) based on 16S ribosomal DNA sequences. Insect Mol Biol 1: 25-36.

Breeuwer, J.A.J. and Werren, J.H. (1990) Cytoplasmic incompatibility and bacterial density in Nasonia vitripennis. Genetics 135: 565-574.

Brower, A.V.Z. (1994) Rapid morpological radiation and convergence among races of the butterfly Heliconius errato inferred from patterns of mitochondrial DNA evolution. Proc Natl Acad Sci USA 91: 6491-6495.

Callaini, G., Dallai, R. and Riparbelli, M.G. (1997) Wolbachiainduced delay of paternal chromatin condensation does not prevent maternal chromosomes from entering anaphase in incompatible crosses of Drosophila simulans. J Cell Sci $\mathbf{1 1 0}$ 271-280.

Caspari, E. and Watson, G.S. (1959) On the evolutionary importance of cytoplasmic sterility in mosquitoes. Evolution 13: $568-570$.

Clancy, D.J. and Hoffman, A.A. (1996) Cytoplasmic incompatibility in Drosophila simulans: Evolving complexity. Trends Ecol Evol 11: 145-146.

Crozier, R.H. (1971) Heterozygosity and sex determination in haplodiploidy. Am Nat 105: 399-412.

Dobson, S.L., Bourtzis, K., Braig, H.R., Jones, B.F., Zhou, W., Rousset, F. and O'Neill, S.L. (1999) Wolbachia infections are distributed throughout insect somatic and germ line tissues. Insect Biochem Mol Biol 29: 153-160.

Fine, P.E.M. (1978) On the dynamics of symbiote-dependent cytoplasmic incompatibility in Culicine mosquitoes. $J$ Invertebr Pathol 30: 10-18.

Goetsch, W. (1953) Vergleichende Biologie der Insektenstaaten. Geest and Portig, Leipzig.

Guillemaud, T., Pasteur, N. and Rousset, F. (1997) Contrasting levels of variability between cytoplasmic genomes and incompatibility types in the mosquito Culex pipiens. Proc $R$ Soc Lond B 264: 245-251.

Hoffmann, A.A. (1988) Partial cytoplasmic incompatibility between two Australian populations of Drosophila melanogaster. Entomologia Expis Applicata 48: 61-67.

Hoffmann, A.A., Clancy, D.J. and Duncan, J. (1996) Naturally occurring Wolbachia infection in Drosophila simulans that does not cause cytoplasmic incompatibility. Heredity 76: 1-8.

Hoffmann, A.A., Clancy, D.J. and Merton, E. (1994) Cytoplasmic incompatibility in Australian populations of Drosophila melanogaster. Genetics 136: 993-999.

Hoffmann, A.A., Hercus, M. and Dagher, H. (1998) Population dynamics of the Wolbachia infection causing cytoplasmic incompatibility in Drosophila melanogaster. Genetics 148: 221-231.

Hoffmann, A.A. and Turelli, M. (1997) Cytoplasmic incompatibility in insects. In: Influential Passengers: Inherited Microorganisms and Arthropod Reproduction (S.L. O'Neill, A.A. Hoffmann and J.H. Werren, eds), pp. 42-80. Oxford University Press, New York.

Hoffmann, A.A., Turelli, M. and Harshman, L.G. (1990) Factors affecting the distribution of cytoplasmic incompatibility in Drosophila simulans. Genetics 126: 933-948.

Hoffmann, A.A., Turelli, M. and Simmons, G.M. (1986) Unidirectional incompatibility between populations of Drosophila simulans. Evolution 40: 692-701.

Hurst, G.D.D., Jiggins, F.M., von der Schulenburg, J.H.G., Bertrand, D., West, S.A., Goriacheva, I.I., Zakharov, I.A., Werren, J.H., Stouthamer, R. and Majerus, M.E.N. (1999) Male-killing Wolbachia in two species of insect. Proc $R$ Soc Lond B 266: 735-740.

Lassy, C.W. and Karr, T.L. (1996) Cytological analysis of fertilization and embryonic development in incompatible crosses of Drosophila simulans. Mech Dev 57: 47-58.

Lofgren, C.S. (1986) History of imported fire ants in the United States. Fire Ants and Leaf Cutting Ants: Biology and Management (C.S., Lofgren and Vander Meer, R.K., eds), pp. 3-49. Westview Press, Boulder, Colorado.

Lofgren, C.S., Banks, W.A. and Glancey, B.M. (1975) Biology and control of imported fire ants. Annu Rev Entomol 20: 130.

van Meer, M.M.M., Witteveldt, J. and Stouthamer, R. (1999) Phylogeny of the arthropod endosymbiont Wolbachia based on the wsp gene. Insect Mol Biol 8: 399-408.

Merçot, H. and Poinsot, D. (1998) ... And discovered on Mount Kilimanjaro. Nature 391: 853.

Min, K.-T. and Benzer, S. (1998) Wolbachia, normally a symbiont of Drosophila, can be virulent, causing degeneration and early death. Proc Natl Acad Sci USA 94: 10 792-10 796

O'Neill, S.L., Giordano, R., Colbert, A.M.E., Karr, T.L. and Robertson, H.M. (1992) 16S rRNA phylogenetic analysis of the bacterial endosymbionts associated with cytoplasmic incompatibility in insects. Proc Natl Acad Sci USA 89: 26992702.

Patterson, R.S. (1994) Biological control of introduced ant species. In: Exotic Ants: Biology, Impact, and Control of Introduced Species (D.F. Williams, ed.), pp. 293-308. Westview Press, Boulder, Colorado.

Porter, S.D., Fowler, H.G. and MacKay, W.P. (1992) Fire ant mound densities in the United States and Brazil (Hymenoptera: Formicidae). J Econ Entomol 85: 1154-1161.

Rieseberg, L.H., Choi, H.C. and Ham, D. (1991) Differential cytoplasmic versus nuclear introgression in Helianthus. $J$ Hered 82: $489-493$.

Ross, K.G. (1992) Strong selection on a gene that influences reproductive competition in a social insect. Nature 355 : $347-$ 349.

Ross, K.G. (1997) Multilocus evolution in fire ants: Effects of selection, gene flow and recombination. Genetics 145: 961-974.

Ross, K.G. and Fletcher, D.J.C. (1985) Genetic origin of male diploidy in the fire ant, Solenopsis invicta (Hymenoptera: Formicidae), and its evolutionary significance. Evolution 39: 888-903. 
Ross, K.G. and Fletcher, D.J.C. (1986) Diploid male production a significant colony mortality factor in the fire ant Solenopsis invicta (Hymenoptera: Formicidae). Behav Ecol Sociobiol 19: 283-291.

Ross, K.G., Krieger, M.J.B., Shoemaker, D.D., Vargo, E.L. and Keller, L. (1997) Hierarchical analysis of genetic structure in native fire ant populations: Results from three classes of molecular markers. Genetics 147: 643-655.

Ross, K.G. and Shoemaker, D.D. (1993) An unusual pattern of gene flow between the two social forms of the fire ant Solenopsis invicta. Evolution 47: 1595-1605.

Ross, K.G. and Shoemaker, D.D. (1997) Nuclear and mitochondrial genetic structure in two social forms of the fire ant Solenopsis invicta: insights into transitions to an alternate social organization. Heredity 78: 590-602.

Ross, K.G., Shoemaker, D.D., Krieger, M.J.B., DeHeer, C.J. and Keller, L. (1999) Assessing genetic structure with multiple classes of markers: a case study involving the introduced fire ant Solenopsis invicta. Mol Biol Evol 16: 525-543.

Ross, K.G. and Trager, J.C. (1990) Systematics and population genetics of fire ants (Solenopsis saevissima complex) from Argentina. Evolution 44: 2113-2134.

Ross, K.G., Vander Meer, R.K., Fletcher, D.J.C. and Vargo, E.L. (1987) Biochemical phenotypic and genetic studies of two introduced fire ants and their hybrid (Hymenoptera: Formicidae). Evolution 41: 280-293.

Ross, K.G., Vargo, E.L. and Fletcher, D.J.C. (1988) Colony genetic structure and queen mating frequency in fire ants of the subgenus Solenopsis (Hymenoptera: Formicidae). Biol $J$ Linn Soc 34: 105-117.

Ross, K.G., Vargo, E.L. and Keller, L. (1996a) Simple genetic basis for important social traits in the fire ant Solenopsis invicta. Evolution 50: 2387-2399.

Ross, K.G., Vargo, E.L. and Keller, L. (1996b) Social evolution in a new environment: The case of introduced fire ants. Proc Natl Acad Sci USA 93: 3021-3025.

Ross, K.G., Vargo, E.L., Keller, L. and Trager, J.C. (1993) Effect of a founder event on variation in the genetic sex-determining system of the fire ant Solenopsis invicta. Genetics 135: 843-854.

Rousset, F., Raymond, M. and Kjellberg, F. (1991) Cytoplasmic incompatibilities in the mosquito Culex pipiens: How to explain a cytotype polymorphism? J Evol Biology 4: 69-81.

Schulenburg, H.G., Hurst, G.D.D., Huigens, T.M.E., van Meer, M.M.M., Jiggins, F.M. and Majerus, M.E.N. (2000) Molecular evolution and phylogenetic utility of Wolbachia fts $Z$ and wsp gene sequences with special reference to the origin of male-killing. Mol Biol Evol 17: 584-600.

Shoemaker, D.D., Katju, V. and Jaenike, J. (1999) Wolbachia and the evolution of reproductive isolation between Drosophila recens and Drosophila subquinaria. Evolution 53: 1157-1164.

Shoemaker, D.D. and Ross, K.G. (1996) Effects of social organization on gene flow in the fire ant Solenopsis invicta. Nature 383: 613-616.

Shoemaker, D.D., Ross, K.G. and Arnold, M.L. (1994) Development of RAPD markers in two introduced fire ants, Solenopsis invicta and S. richteri, and their application to the study of a hybrid zone. Mol Ecol 3: 531-539.

Shoemaker, D.D., Ross, K.G. and Arnold, M.L. (1996) Genetic structure and evolution of a fire ant hybrid zone. Evolution 50: 1958-1976.
Simon, C., Frati, F., Beckenbach, A., Crespi, B., Liu, H. and Flook, P. (1994) Evolution, weighting, and phylogenetic utility of mitochondrial gene sequences and a compilation of conserved polymerase chain reaction primers. Ann Entomol Soc 81: 651-701.

Sinkins, S.P., Braig, H.R. and O'Neill, S.L. (1995a) Wolbachia pipientis: Bacterial density and unidirectional cytoplasmic incompatibility between infected populations of Aedes albopictus. Exp Parasitol 81: 284-291.

Sinkins, S.P., Braig, H.R. and O'Neill, S.L. (1995b) Wolbachia superinfections and the expression of cytoplasmic incompatibility. Proc R Soc Lond B 261: 325-330.

Sinkins, S.P., Curtis, C.F. and O'Neill, S.L. (1997) The potential application of inherited symbiont systems to pest control. In: Influential Passengers: Inherited Microorganisms and Arthropod Reproduction (S.L. O'Neill, A.A. Hoffmann and J.H. Werren, eds), pp. 155-175. Oxford University Press, New York.

Sironi, M., Bandi, C., Sacchi, L., Di Sacco, B., Damiani, G. and Genchi, C. (1995) Molecular evidence for a close relative of the arthropod endosymbiont Wolbachia in a filarial worm. Mol Biochem Parasitol 74: 223-227.

Solignac, M., Vautrin, D. and Rousset, F. (1994) Widespread occurrence of the proteobacteria Wolbachia and partial cytoplasmic incompatibility in Drosophila melanogaster. C R Acad Sci Paris 317: 461-470.

Stouthamer, R. (1997) Wolbachia-induced parthenogenesis. In: Influential Passengers: Inherited Microorganisms and Arthropod Reproduction (S.L., O'Neill, A.A., Hoffmann and Werren, J.H., eds), pp. 102-124. Oxford University Press, New York.

Stouthamer, R., Breeuwer, J.A.J. and Hurst, G.D.D. (1999) Wolbachia pipientis: Microbial manipulator of arthropod reproduction. Annu Rev Microbiol 53: 71-102.

Stouthamer, R., Breeuwer, J.A.J., Luck, R.F. and Werren, J.H. (1993) Molecular identification of microorganisms associated with parthenogenesis. Nature 361: 66-68.

Swofford, D.L. (1999) PAUP*. Phylogenetic Analysis Using Parsimony ("${ }^{*}$ and other methods), Version 4.0.

Trager, J.C. (1991) A revision of the fire ants, Solenopsis geminata group (Hymenoptera: Formicidae: Myrmicinae). J New York Entomol Soc 99: 141-198.

Turelli, M. (1994) Evolution of incompatibility-inducing microbes and their hosts. Evolution 48: 1500-1513.

Turelli, M. and Hoffmann, A.A. (1991) Rapid spread of an inherited incompatibility factor in California. Nature 353: 440-442.

Turelli, M. and Hoffmann, A.A. (1995) Cytoplasmic incompatibility in Drosophila simulans: Dynamics and parameter estimates from natural populations. Genetics 140: 1319-1338.

Turelli, M. and Hoffmann, A.A. (1999) Microbe-induced cytoplasmic incompatibility as a mechanism for introducing transgenes into arthropod populations. Insect Mol Biol 8: 243-255.

Vavre, F., Fleury, F., Lepetit, D., Fouillet, P. and Bouletreau (1999a) Phylogenetic evidence for horizontal transmission of Wolbachia in host-parasitoid associations. Mol Biol Evol 16: 1711-1723.

Vavre, F., Fleury, F., Varaldi, J., Fouillet, P. and Bouletreau, M. (2000) Evidence for female mortality in Wolbachia-mediated cytoplasmic incompatibility in haplodiploid insects: Epidemiologic and evolutionary consequences. Evolution 54: 191-200.

Vavre, F., Girin, C. and Bouletreau, M. (1999b) Phylogenetic status of a fecundity-enhancing Wolbachia that does not induce thelytoky in Trichogramma. Insect Mol Biol 8: 67-72. 
Venanzetti, F., Cesaroni, D., Mariottini, P. and Sbordoni, V. (1993) Molecular phylogenies in Dolichopoda cave criquets and mtDNA rate calibration. Mol Phylogenet Evol 2: 275-280.

Wade, M.J. and Chang, N.W. (1995) Increased male-fertility in Tribolium confusum beetles after injection with the intracellular parasite Wolbachia. Nature 51: 72-74.

Werren, J.H. (1997) Biology of Wolbachia. Annu Rev Entomol 42: $537-609$.

Werren, J.H. (1998) Wolbachia and speciation. In: Endless Forms: Species and Speciation (Howard, D. and Berlocher, S., eds), pp. 245-260. Oxford University Press, New York.

Werren, J.H., Guo, L. and Windsor, D.W. (1995a) Distribution of Wolbachia in neotropical arthropods. Proc $R$ Soc Lond $B$ 262: 197-204.
Werren, J.H. and O'Neill, S.L. (1997) The evolution of heritable symbionts. In: Influential Passengers: Inherited Microorganisms and Arthropod Reproduction. (O'Neill, S.L. Hoffmann, A.A. and Werren, J.H., eds), pp. 1-41. Oxford University Press, New York.

Werren, J.H. and Windsor, D.M. (2000) Wolbachia infection frequencies in insects: Evidence of a global equilibrium? Proceedings of the $R$ Soc Lond B 267.

Werren, J.H., Zhang, W. and Guo, L.R. (1995b) Evolution and phylogeny of Wolbachia: Reproductive parasites of arthropods. Proc R Soc Lond B 261: 55-71.

Zhou, W., Rousset, F. and O'Neill, S.L. (1998) Phylogeny and PCR-based classification of Wolbachia strains using wsp gene sequences. Proc $R$ Soc Lond B 265: 509-515. 